\title{
RADEMACHER-TYPE FORMULAS FOR THE MULTIPLICITIES OF IRREDUCIBLE HIGHEST-WEIGHT REPRESENTATIONS OF AFFINE LIE ALGEBRAS
}

\section{J. MORENO AND A. ROCHA-CARIDI}

The weight lattice of an integrable irreducible highest-weight representation of an affine Lie algebra is a union of infinite strings. Furthermore, the multiplicities in a finite number of strings determine all the multiplicities. Kac and Peterson $[1,2]$ have shown that the multiplicities in the same string are the Fourier coefficients of a modular form of negative weight, called a string function. This result, together with combinatorial identities for the Dedekind $\eta$-function, was successfully used in [1 and 2] to determine the string functions in many interesting cases. In this paper, we make use of the result of [1 and 2] to adapt Rademacher's circle method to the string functions and derive formulas for their coefficients. The formulas obtained are of the type proved by Rademacher in [3] for the partition function. This new approach opens the way towards an explicit determination of the Fourier coefficients which goes beyond the combinatorial method. As an example, we show how to calculate the coefficients in the case of the affine Lie algebra of type $C_{4}^{(1)}$, which is the simplest nontrivial case not treated in [1 and 2].

1. String functions. Our main purpose here is to state a version of the transformation law for string functions obtained by Kac and Peterson [1, 2]. We refer the reader to the original papers for details.

Let $\overline{\mathfrak{g}}$ be a complex simple Lie algebra. Let $\overline{\mathfrak{h}}$ be a Cartan subalgebra of $\overline{\mathfrak{g}}$ and denote by $\bar{\Delta}$ the root system of $(\overline{\mathfrak{g}}, \overline{\mathfrak{h}})$. Then $\overline{\mathfrak{g}}=\overline{\mathfrak{h}} \oplus\left(\bigoplus_{\alpha \in \bar{\Delta}} \overline{\mathfrak{g}}_{\alpha}\right)$, where $\overline{\mathfrak{g}}_{\alpha}=\{X \in \overline{\mathfrak{g}} \mid[H, X]=\alpha(H) X$ for all $H \in \overline{\mathfrak{h}}\}$. We fix a set of positive roots $\bar{\Delta}_{+}$with simple roots $\alpha_{1}, \ldots, \alpha_{l}$. Let $\theta$ be the highest root of $\bar{\Delta}_{+}$. Let $(X, Y)=2 g B(X, Y)$ for all $X, Y \in \overline{\mathfrak{g}}$, where $B$ is the Killing form of $\overline{\mathfrak{g}}$ and $g=(B(\theta, \theta))^{-1}$.

We denote by $\mathfrak{g}$ the (untwisted) affine Lie algebra associated with $\overline{\mathfrak{g}}$. That is, $\mathfrak{g}=\left(\mathbf{C}\left[t, t^{-1}\right] \otimes \overline{\mathfrak{g}}\right) \oplus \mathbf{C} c \oplus \mathbf{C} d$, where $c$ is central and we define

$$
\begin{gathered}
{[P \otimes X, Q \otimes Y]=P Q \otimes[X, Y]+\operatorname{Res}\left(\frac{d P}{d t} Q\right)(X, Y) c,} \\
{[d, P \otimes X]=t \frac{d P}{d t} \otimes X, \quad \text { and }[d, d]=0,}
\end{gathered}
$$

for all $P, Q \in \mathbf{C}\left[t, t^{-1}\right], X, Y \in \overline{\mathfrak{g}} . \mathfrak{h}=\overline{\mathfrak{h}} \oplus \mathbf{C} c \oplus \mathbf{C} d$ is the Cartan subalgebra of $\mathfrak{g}$. We extend $($,$) to \mathfrak{h} \times \mathfrak{h}$ by setting $(H, c)=(H, d)=0$ for all $H \in \mathfrak{h}$,

\footnotetext{
Received by the editors September 27, 1986 and, in revised form, November 30, 1986.

1980 Mathematics Subject Classification (1985 Revision). Primary 17B65, 17B67, $10 \mathrm{D} 12$. 
$(c, d)=1$ and $(c, c)=(d, d)=0$. We also denote by $($,$) the form induced on$ $\mathfrak{h}^{*} \times \mathfrak{h}^{*}$.

Let $\mathfrak{n}_{+}=\overline{\mathfrak{n}}_{+} \oplus(t \mathbf{C}[t] \otimes \overline{\mathfrak{g}})$, where $\overline{\mathfrak{n}}_{+}=\bigoplus_{\alpha \in \bar{\Delta}_{+}} \overline{\mathfrak{g}}_{\alpha}$. For each $\Lambda \in \mathfrak{h}^{*}$, let $L(\Lambda)$ be the irreducible highest-weight representation of $\mathfrak{g}$ with highest weight $\Lambda . L(\Lambda)$ is the unique irreducible representation with the property that there exists $v \in L(\Lambda)$ such that $L(\Lambda)=\mathrm{U}(\mathfrak{g}) v, \mathfrak{n}_{+} v=0$, and $H v=\Lambda(H) v$ for all $H \in \mathfrak{h}$. Here, $\mathrm{U}(\mathfrak{g})$ is the universal enveloping algebra of $\mathfrak{g}$. One has

$$
L(\Lambda)=\bigoplus_{\lambda \in P(\Lambda)} L(\Lambda)_{\lambda},
$$

where $L(\Lambda)_{\lambda}=\{v \in L(\Lambda) \mid H v=\lambda(H) v$, for all $H \in \mathfrak{h}\}$ and $P(\Lambda)=\{\lambda \in$ $\left.\mathfrak{h}^{*} \mid L(\lambda)_{\lambda} \neq(0)\right\}$. We set $\operatorname{mult}_{\Lambda}(\lambda)=\operatorname{dim} L(\Lambda)_{\lambda}$.

Let $\delta \in \mathfrak{h}^{*}$ be such that $\delta(\overline{\mathfrak{h}} \oplus \mathbf{C} c)=0$ and $\delta(d)=1$, and put $\alpha_{0}=\delta-\theta$. Let $P$ (resp. $P_{+}$) be the set of all $\lambda \in \mathfrak{h}^{*}$ such that

$$
2 \frac{\left(\lambda, \alpha_{i}\right)}{\left(\alpha_{i}, \alpha_{i}\right)} \in \mathbf{Z} \quad\left(\text { resp. } \mathbf{Z}_{+}\right) \quad \text { for } i=0,1, \ldots, l \text {. }
$$

Let $\Lambda$ be an arbitrary but fixed element of $P_{+}$. Then $m=\Lambda(c)$ is a nonnegative integer called the level of $L(\Lambda)$. Assume that $m>0$, i.e., $\operatorname{dim} L(\Lambda)>1$. Let $\rho \in \mathfrak{h}^{*}$ be defined by $\left(\rho, \alpha_{i}\right)=\frac{1}{2}\left(\alpha_{i}, \alpha_{i}\right), i=0, \ldots, l$, and $\rho(d)=0$. Let $\lambda \in P$. If $\lambda-n \delta \notin P(\Lambda)$ for all $n \in \mathbf{Z}$, then set $C_{\lambda}^{\Lambda}=0$. Otherwise, let $n_{0}$ be minimal so that $\lambda_{0}=\lambda-n_{0} \delta \in P(\Lambda)$, and set

$$
C_{\lambda}^{\Lambda}=e^{-s_{\Lambda}\left(\lambda_{0}\right) \delta} \sum_{n \geq 0} \operatorname{mult}_{\Lambda}\left(\lambda_{0}-n \delta\right) e^{-n \delta},
$$

where

$$
s_{\Lambda}\left(\lambda_{0}\right)=\frac{|\Lambda+\rho|^{2}}{2(m+g)}-\frac{|\rho|^{2}}{2 g}-\frac{\left|\lambda_{0}\right|^{2}}{2 m} .
$$

Set $\delta=-2 \pi i \tau$. Then, viewed as a function of $\tau, C_{\lambda}^{\Lambda}$ is a complex-valued function on $H=\{\tau \in \mathbf{C} \mid \operatorname{Im} \tau>0\}$, called the string function of $\lambda$, which has at most poles as singularities $[\mathbf{1}, \mathbf{2}]$.

Let $M$ be the Z-span of $\bar{W} \theta$, when $\bar{W}$ is the Weyl group of $\overline{\mathfrak{g}}$. Given $\mu \in \mathfrak{h}^{*}$, let $\bar{\mu}$ denote the restriction of $\mu$ to $\overline{\mathfrak{h}}^{*}$. Let $\bar{P}=\{\bar{\mu} \mid \mu \in P\}$. Let $\lambda$ be an arbitrary but fixed element of $P$.

THEOREM $1.1[\mathbf{1}, 2]$.

$$
C_{\lambda}^{\Lambda}\left(-\frac{1}{\tau}\right)=\left(\frac{\tau}{i}\right)^{-l / 2} \sum_{\Lambda^{\prime}, \lambda^{\prime}} b\left(\Lambda^{\prime}, \lambda^{\prime}\right) C_{\lambda^{\prime}}^{\Lambda^{\prime}}(\tau),
$$

where

$$
\begin{array}{r}
b\left(\Lambda^{\prime}, \lambda^{\prime}\right)=i^{\left|\bar{\Lambda}_{+}\right|}|\bar{P} / M|^{-1}(m(m+g))^{-l / 2} \exp \left(2 \pi i \frac{\left(\bar{\lambda}, \bar{\lambda}^{\prime}\right)}{m}\right) \\
\cdot \sum_{w \in \bar{W}}(\operatorname{det} w) \exp \left(-2 \pi i \frac{\left(\bar{\Lambda}+\bar{\rho}, w\left(\bar{\Lambda}^{\prime}+\bar{\rho}\right)\right)}{m+g}\right),
\end{array}
$$


and the sum is over all $\Lambda^{\prime} \in P_{+} / \mathbf{C} \delta$ and $\lambda^{\prime} \in P /(m M+\mathbf{C} \delta)$ with $\Lambda^{\prime}(c)=$ $\lambda^{\prime}(c)=m$.

Furthermore, if $s_{\Lambda}(\lambda)=\left[s_{\Lambda}(\lambda)\right]+\alpha$, with $0 \leq \alpha<1$, then

$$
C_{\lambda}^{\Lambda}(\tau+1)=e^{2 \pi i \alpha} C_{\lambda}^{\Lambda}(\tau)
$$

Given $\sigma=\left(\begin{array}{l}a b \\ c d\end{array}\right) \in \operatorname{SL}(2, \mathbf{Z})$, one defines $\sigma(\tau)=(a \tau+b) /(c \tau+d)$ for all $\tau \in H$. We denote by $\Gamma$ the group $\mathrm{SL}(2, \mathbf{Z})$ viewed as a group acting on $H$ in this way. Since every $\sigma \in \Gamma$ is a finite product of the elements: $\tau \mapsto-1 / \tau$ and $\tau \mapsto \tau+1$, we deduce, from Theorem 1.1, the following.

COROLlaRY 1.2. Let $\sigma \in \Gamma$. Then

$$
C_{\lambda}^{\Lambda}(\sigma(\tau))=\left(\frac{c \tau+d}{i}\right)^{-l / 2} \sum_{\Lambda^{\prime}, \lambda^{\prime}} b_{\sigma}\left(\Lambda^{\prime}, \lambda^{\prime}\right) C_{\lambda^{\prime}}^{\Lambda^{\prime}}(\tau),
$$

where the sum is as in Theorem 1.1 and $b_{\sigma}\left(\Lambda^{\prime}, \lambda^{\prime}\right) \in \mathbf{C}$.

2. The Rademacher formula. Throughout this section we fix a pair $\Lambda \in$ $\mathrm{P}_{+}$and $\lambda \in \mathrm{P}$ and denote the corresponding string function by $C(\tau)$. For a pair $\mathcal{L}=\left(\Lambda^{\prime}, \lambda^{\prime}\right)$ as in Corollary 1.2, with $\Lambda \in \mathrm{P}_{+} \bmod \mathbf{C} \delta, \lambda^{\prime} \in \mathrm{P} \bmod (m M+$ $\mathbf{C} \delta)$ and $\Lambda^{\prime}(c)=\lambda^{\prime}(c)=m$, we let $C^{\prime}(\tau)$ denote the string function $C_{\lambda^{\prime}}^{\Lambda^{\prime}}(\tau)$, $\alpha^{\prime}$ the exponent $0 \leq \alpha^{\prime}<1$ which makes $e^{-2 \pi i \alpha^{\prime} \tau} C^{\prime}(\tau)$ periodic, $\mu^{\prime}$ the order of the pole of $e^{-2 \pi i \alpha^{\prime} \tau} C^{\prime}(\tau)$ at infinity and $a_{\nu}^{\prime}=a_{\nu}^{\prime}(\mathcal{L}),-\mu^{\prime} \leq \nu \leq-1$, the coefficients of the polar part of $C^{\prime}(\tau)$. Let $k$ be a positive integer and for any integer $h$ with $1 \leq h \leq k,(h, k)=1$, let $\sigma$ denote the unimodular matrix

$$
\left(\begin{array}{cc}
h & -\frac{h k^{\prime}+1}{k} \\
k & -h^{\prime}
\end{array}\right)
$$

where $h h^{\prime}+1 \equiv 0 \bmod k$. For integers $k, n, \nu$, and a pair $\mathcal{L}=\left(\Lambda^{\prime}, \lambda^{\prime}\right)$, define the exponential sum

$$
A_{k}(\mathcal{L}, n, \nu)=\sum_{\substack{h \bmod k \\(h, k)=1}} b_{\sigma}(\mathcal{L}) e^{2 \pi i\left\{\left(\alpha^{\prime}+\nu\right) h^{\prime}-(\alpha+n) h\right\} / k},
$$

where $b_{\sigma}(\mathcal{L})=b_{\sigma}\left(\Lambda^{\prime}, \lambda^{\prime}\right)$ are the coefficients of Corollary 1.2. For a positive real number $y$ and $s$ any complex number, we let

$$
L_{s}(y)=\sum_{q=0}^{\infty} \frac{y^{q}}{\Gamma(1+s+q) q !}
$$

denote the modified Bessel function of order $s$. The main new result is the following:

THEOREM 2.1. With notations as above, we have for the nth Fourier coefficient of the string function $C_{\lambda}^{\Lambda}(\tau)$ the representation

$$
\begin{aligned}
& \operatorname{mult}_{\Lambda}(\lambda-(\mu+n) \delta)=\sum_{k=1}^{\infty} \sum_{\mathcal{L}} \sum_{\nu=-\mu^{\prime}}^{-1} A_{k}(\mathcal{L}, n, \nu) a_{\nu}^{\prime}(\mathcal{L}) \\
& \cdot k^{-2-l / 2}(2 \pi)^{2+l / 2}\left(-\alpha^{\prime}-\nu\right)^{1+l / 2} L_{1+l / 2}\left(\frac{4 \pi^{2}}{k^{2}}(\alpha+n)\left(-\alpha^{\prime}-\nu\right)\right)
\end{aligned}
$$


where the sum $\sum_{\mathcal{L}}$ is taken over all distinct pairs $\mathcal{L}=\left(\Lambda^{\prime}, \lambda^{\prime}\right)$ with $\Lambda \in$ $\mathrm{P}_{+} \bmod \mathbf{C} \delta, \lambda^{\prime} \in \mathbf{P} \bmod (m M+\mathbf{C} \delta)$, and $\Lambda^{\prime}(c)=\lambda^{\prime}(c)=m$.

REMARKS. 1. As in Rademacher's derivation of the analogous formula for the partition function [3, p. 292] the proof uses the idea of Ford circles based on the Farey fractions to refine the circle method. Since the string functions are not modular forms for the full unimodular group in the sense of the classical definition, the method has to be adapted so that the transformation formula of Corollary 1.2 can be used.

2. An interesting byproduct of the proof is the appearance of the Kloostermann-like sum $A_{k}(\mathcal{L}, n, \nu)$ with its dependence on the coefficients $b_{\sigma}$ which by Corollary 1.2 are the matrix coefficients of a finite-dimensional representation of the group $\Gamma / \Gamma\left(N^{*}\right)$ for a suitable $N^{*}$ (made explicit in the proof of Theorem $1.1[2])$.

3. Formula 2.2 implies that the multiplicities that appear in the polar part of a string function determine all other multiplicities.

4. The Rademacher formula of Theorem 2.1 is useful in the study of the asymptotic behavior of the coefficients $\operatorname{mult}_{\Lambda}(\lambda-(\mu+n) \delta)$ and in this sense the major result can be thought of as an elaboration of the asymptotic estimates of $\mathrm{Kac}$ and Peterson [2, §4.7, Theorem B].

3. Examples. 1. Let $\Delta$ be the root system given by the Dynkin diagram of type $A_{1}^{(1)}$.

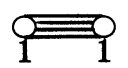

At level one, there are two string functions $C_{\Lambda_{0}}^{\Lambda_{0}}=C_{\Lambda_{1}}^{\Lambda_{1}}$ with characteristic $S_{\Lambda_{0}}^{\Lambda_{0}}=-1 / 24[1,2]$. In this case $\alpha=23 / 24$ and $u=1$. By Corollary 1.2 we have

$$
C_{\Lambda_{0}}^{\Lambda_{0}}(\sigma(\tau))=\left(\frac{(\tau+d)}{i}\right)^{-1 / 2} b_{\sigma}(\mathcal{L}) C_{\Lambda_{0}}^{\Lambda_{0}}(\tau)
$$

where $b_{\sigma}(\mathcal{L})^{-24}=1[1,2]$. Formula $(2.2)$ reduces to

$$
\begin{aligned}
\operatorname{mult}_{\Lambda_{0}}\left(\Lambda_{0}-n \delta\right)= & 2 \pi\left(\frac{\pi}{12}\right)^{3 / 2} \sum_{k=1}^{\infty} A_{k}(\mathcal{L}, n-1,-1) k^{-5 / 2} \\
& \cdot L_{3 / 2}\left(\left(\frac{\pi}{12 k}\right)^{2}(2 h n-1)\right)
\end{aligned}
$$

with

$$
A_{k}(\mathcal{L}, n-1,-1)=\sum_{\substack{h \bmod k \\(h, k)=1}} b_{\sigma}(\mathcal{L}) e^{(\pi i / 12)\left(h-h^{\prime}\right)} e^{-2 \pi i h n / k}
$$

This is precisely Rademacher's formula for $p(n)[3, \mathrm{p} .292]$.

2. Let $\Delta$ be the root system given by the Dynkin diagram of type $C_{4}^{(1)}$ :

$$
\mathrm{O} \Rightarrow \mathrm{Q}-\mathrm{O}=\mathrm{O}
$$


There are seven distinct string functions for the representations of level one, namely:

$$
\begin{gathered}
C_{\Lambda_{0}}^{\Lambda_{0}}=C_{\Lambda_{4}}^{\Lambda_{4}}, \quad C_{\Lambda_{2}-\delta}^{\Lambda_{0}}=C_{\Lambda_{2}}^{\Lambda_{4}}, \quad C_{\Lambda_{4}-2 \delta}^{\Lambda_{0}}=C_{\Lambda_{0}}^{\Lambda_{4}}, \quad C_{\Lambda_{1}}^{\Lambda_{1}}=C_{\Lambda_{3}}^{\Lambda_{3}}, \\
C_{\Lambda_{3}-\delta}^{\Lambda_{1}}=C_{\Lambda_{1}}^{\Lambda_{3}}, \quad C_{\Lambda_{0}}^{\Lambda_{2}}=C_{\Lambda_{4}-\delta}^{\Lambda_{2}}, \quad \text { and } C_{\Lambda_{2}}^{\Lambda_{2}} .
\end{gathered}
$$

In Table 1 we list the characteristic $s=s_{\Lambda}(\lambda)$ of $C_{\lambda}^{\Lambda}$, the exponent of the periodicity factor $\alpha=\alpha(\Lambda, \lambda)$ and the order of the pole $u=u(\Lambda, \lambda)$ of $e^{-2 \pi i \alpha \tau} C_{\lambda}^{\Lambda}(\tau)$

\section{TABLE 1}

\begin{tabular}{lcrrr}
\hline$\Lambda$ & $\lambda$ & \multicolumn{1}{c}{$s$} & $\alpha$ & $u$ \\
\hline$\Lambda_{0}$ & $\Lambda_{0}$ & $-\frac{1}{4}$ & $\frac{3}{4}$ & 1 \\
$\Lambda_{0}$ & $\Lambda_{2}-\delta$ & $\frac{1}{4}$ & $\frac{1}{4}$ & 0 \\
$\Lambda_{0}$ & $\Lambda_{4-2 \delta}$ & $\frac{3}{4}$ & $\frac{3}{4}$ & 0 \\
$\Lambda_{1}$ & $\Lambda_{1}$ & $-\frac{1}{8}$ & $\frac{7}{8}$ & 1 \\
$\Lambda_{1}$ & $\Lambda_{3}-\delta$ & $\frac{3}{8}$ & $\frac{3}{8}$ & 0 \\
$\Lambda_{2}$ & $\Lambda_{0}$ & $\frac{5}{12}$ & $\frac{5}{12}$ & 0 \\
$\Lambda_{2}$ & $\Lambda_{2}$ & $-\frac{1}{12}$ & $\frac{11}{12}$ & 1
\end{tabular}

Using the fact that only $C_{\Lambda_{0}}^{\Lambda_{0}}, C_{\Lambda_{1}}^{\Lambda_{1}}$, and $C_{\Lambda_{2}}^{\Lambda_{2}}$ have nontrivial polar parts, the Rademacher formula reduces to

$$
\begin{aligned}
\operatorname{mult}_{\Lambda}(\lambda-(n+1) \delta)= & \sum_{k=1}^{\infty} \sum_{\mathcal{L}} A_{k}(\mathcal{L}, n,-1) k^{-4}\left(1-\alpha^{\prime}\right)^{-1} \\
& \cdot L_{-1}\left(\frac{4 \pi^{2}}{k^{2}}(\mathcal{L}+n)\left(1-\alpha^{\prime}\right)\right)
\end{aligned}
$$

where $\mathcal{L}=\left\{\left(\Lambda_{0}, \Lambda_{0}\right),\left(\Lambda_{1}, \Lambda_{1}\right),\left(\Lambda_{2}, \Lambda_{2}\right)\right\}$ and $\alpha^{\prime}$ is $3 / 4,7 / 8$, and $11 / 12$, respectively.

FINAL REMARK. A formula analogous to that of Theorem 2.1 was also obtained in the full generality of affine Lie algebras, including the twisted affine case. In a forthcoming paper we will discuss the $b_{\sigma}$ 's and the case $C_{l}^{(1)}$.

\section{REFERENCES}

1. V. G. Kac and D. H. Peterson, Affine Lie algebras and Hecke modular forms, Bull. Amer. Math. Soc. (N.S.) 3 (1980), 1057-1061.

2. __ Infinite dimensional Lie algebras, theta functions and modular forms, Adv. in Math. 53 (1984), 125-264.

3. H. Rademacher, Topics in analytic number theory, Springer-Verlag, New York, 1973.

Department of Mathematics, Baruch College, C.U.N.Y., NeW York, NEW YORK 10010 